\title{
Theoretical approach to the magnetostructural correlations in the spin-Peierls compound $\mathrm{CuGeO}_{3}$
}

\author{
Eliseo Ruiz, Joan Cano, Santiago Alvarez, and Pere Alemany \\ Departments de Química Inorgànica i Química Física and Centre de Recerca en Química Teòrica (CeRQT), \\ Facultat de Química, Universitat de Barcelona, Diagonal 647, 08028 Barcelona, Spain \\ Michel Verdaguer \\ Laboratoire de Chimie Inorganique et Matériaux Moléculaires, Unité associée au CNRS 7071, Case 42, Batiment F74, \\ Université Pierre et Marie Curie, 4 place Jussieu, F-75252, Paris Cedex 05, France
}

(Received 20 July 1999)

\begin{abstract}
A theoretical density-functional study has been carried out to analyze the exchange coupling in the chains of $\mathrm{CuGeO}_{3}$ using discrete models. The results show a good agreement with the experimental exchange coupling constant $(J)$ together with a strong dependence of $J$ with the $\mathrm{Cu}-\mathrm{O}-\mathrm{Cu}$ angle. The calculation of the $J$ values for a distorted model indicates a larger degree of dimerization than those reported previously.
\end{abstract}

\section{INTRODUCTION}

The spin-Peierls transition is an interesting phenomenon that appears in spin chains. ${ }^{1}$ Below a critical temperature, the chains show a structural distortion and the magnetic susceptibility presents a rapid drop. The first systems known to undergo such a transition were organic materials like TTFCuBDT or MEM(TCNQ $)_{2} \cdot{ }^{2}$ Since Hase et al. discovered in 1993 the inorganic spin-Peierls compound ${ }^{3} \mathrm{CuGeO}_{3}$, a huge amount of work has been devoted to the special behavior of this compound. ${ }^{4}$ Above $14 \mathrm{~K}, \mathrm{CuGeO}_{3}$ has an orthorhombic unit cell (originally the Pbmm space group has been proposed, ${ }^{5-7}$ although recent measurements ${ }^{8}$ suggest $\left.P 2{ }_{1} 2{ }_{1} 2\right)$ with a long-range-order antiferromagnetic coupling. The low-temperature phase also shows an orthorhombic unit cell (Bbcm space group) but slightly distorted to give a "dimer', character to the chains. ${ }^{9}$ Recently, $\alpha-\mathrm{NaV}_{2} \mathrm{O}_{5}$ has been proposed as a possible inorganic spin Peierls compound, ${ }^{10}$ but recent diffraction experiments have introduced some controversy in this respect. ${ }^{11,12}$

The structure of the high-temperature phase of $\mathrm{CuGeO}_{3}$ is schematized in Fig. 1 and the main bonding parameters are summarized in Table I. The structure is built from linear chains of $\mathrm{Cu}^{2+}$ ions with square-planar coordination running along the $c$ direction. The oxygen atoms bridging two copper atoms are simultaneously forming tetrahedral $\mathrm{GeO}_{4}$ units that provide a link between neighbor copper chains in the $b$ direction. The exchange-coupling constant obtained from inelastic neutron-scattering measurements shows a strong antiferromagnetic coupling in the $c$ direction due to the exchange interaction along the copper chains $\left[J_{c} \sim-120 \mathrm{~K}\right.$, see Eq. (1) below]. Non-negligible but small values are found for the interchain coupling along the other crystallographic directions: $J_{b} \sim 0.1 J_{c}$ and $J_{a} \sim-0.01 J_{c} .{ }^{13}$ Substantially higher values of $J_{c}$ are obtained from the observed dispersion for the magnetic excitations $\left(J_{c} \sim-150-170 \mathrm{~K}\right),{ }^{14,15}$ from magnetic susceptibility data $\left(J_{c} \sim-160 \mathrm{~K}\right),{ }^{16}$ or from high-field Faraday rotation experiments $\left(J_{c} \sim-180 \mathrm{~K}\right) .^{17}$
Exchange coupling in the chain direction is usually described with a Heisenberg Hamiltonian with two exchange constants

$$
H=-J \sum_{i}\left[\left(1+\delta(-1)^{i}\right) S_{i} S_{i+1}+\alpha S_{i} S_{i+2}\right]
$$

where $J$ is the exchange constant between neighboring copper atoms (usually this parameter is compared with the experimental $J_{c}$ values), $\alpha J$ is the coupling constant between next-nearest neighbors. In the previous expression $\alpha$ is a frustration parameter whose value has been estimated between 0.24 and $0.36,{ }^{15,16}$ and $\delta$ is the dimerization parameter that vanishes above the critical temperature. A value of 0.012 for $\delta$ reproduces the experimental spin gap of $2.1 \mathrm{meV}$ using a static phonon model. ${ }^{14}$ However, larger $\delta$ values as high as 0.12 are predicted using more sophisticated dynamic spinphonon models to describe the magnetic excitation spectrum. $^{18}$

Although several theoretical studies of the electronic structure in $\mathrm{CuGeO}_{3}$ have been reported, ${ }^{19-22}$ only the paper

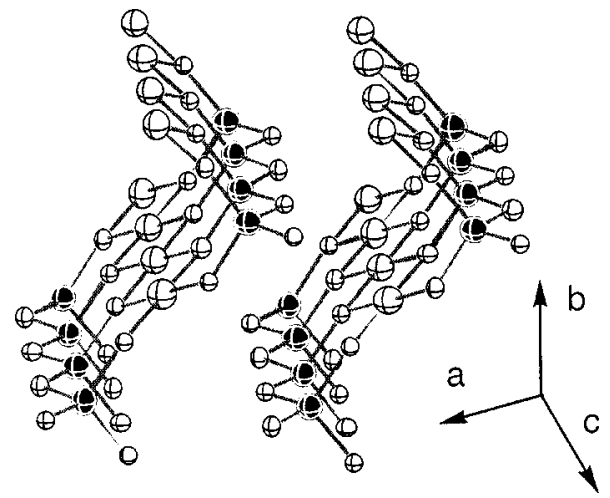

FIG. 1. Structure corresponding to the high-temperature phase of $\mathrm{CuGeO}_{3}$ (Pbmm space group). Small white, large white, and black spheres correspond, respectively, to $\mathrm{O}, \mathrm{Cu}$, and $\mathrm{Ge}$ atoms. 
TABLE I. Main structural parameters corresponding to the two phases of $\mathrm{CuGeO}_{3}$. For the low-temperature phase, each value corresponds to one of $\mathrm{Cu}_{2} \mathrm{O}_{2}$ moieties after the dimerization of the chain structure.

\begin{tabular}{lccc}
\hline \hline & $\begin{array}{c}\text { High-temperature } \\
\text { phase }(\text { Pbmm })\end{array}$ & \multicolumn{2}{c}{$\begin{array}{c}\text { Low-temperature } \\
\text { phase }(\text { Bbcm })\end{array}$} \\
\hline$d(\mathrm{Cu}-\mathrm{O})(\AA)$ & 1.933 & 1.935 & 1.932 \\
$d(\mathrm{Cu}-\mathrm{Cu})(\AA)$ & 2.944 & 2.956 & 2.933 \\
$\mathrm{Cu}-\mathrm{O}-\mathrm{Cu}$ & $99.2^{\circ}$ & $99.6^{\circ}$ & $98.8^{\circ}$ \\
$\mathrm{O}-\mathrm{O}-\mathrm{Ge}$ & $158.8^{\circ}$ & $159.9^{\circ}$ & $158.1^{\circ}$ \\
\hline \hline
\end{tabular}

of Zagoulaev and Tupitsyn is aimed towards a determination of the exchange coupling constants using semiempirical periodic electronic structure calculations. Therefore, the aim of the present paper is to present an $a b$ initio study of the magnetic properties of such compound by analyzing the influence of the structural parameters on the exchange coupling. The knowledge of these magnetostructural correlations can be of great help for the interpretation of the changes in magnetic behavior that appear when the chains are distorted upon cooling the compound below the critical temperature, as well as in the interpretation of the data related to spin-phonon coupling.

\section{RESULTS AND DISCUSSION}

In recent works we have shown the ability of hybrid density-functional methods to provide accurate numerical estimates of the exchange coupling constant $J$ in transitionmetal molecular complexes. ${ }^{23-25}$ For this purpose we use the B3LYP method ${ }^{26}$ as implemented in the GAUSSIAN package ${ }^{27}$ combined with a modified broken-symmetry approach. The B3LYP method is the most popular form of the so-called hybrid functionals. In these methods the exact exchange, calculated using the Kohn-Sham orbitals, is mixed with the pure generalized-gradient approximation (GGA) functional by fitting, in the B3LYP case, three mixing parameters to some set of experimental data. The larger accuracy of the hybrid functionals in comparison with the GGA ones can be easily understood by the reduction of the self-interaction error due to the insertion of the exact exchange. A triple- $\zeta$ basis set is used for the transition metal, ${ }^{28}$ while a double- $\zeta$ basis set is employed for the rest of atoms. ${ }^{29}$ Using this approach we have calculated exchange constants for complete molecular structures obtaining a very good agreement with the available experimental values. This computational approach allows us to analyze the dependence of the exchange coupling on different structural parameters. The study of such magnetostructural correlations contributes to the understanding of the mechanism of exchange coupling and may suggest possible structural modifications to design new compounds with interesting magnetic properties.

Among the families of the transition-metal molecular complexes that we have studied, the hydroxo- and alkoxobridged copper (II) binuclear complexes are those that present structural similarities with $\mathrm{CuGeO}_{3}$. From an experimental point of view, the well-known magnetostructural correlation proposed by Hodgson and Hatfield for the hydroxobridged copper (II) complexes shows a strong dependence of
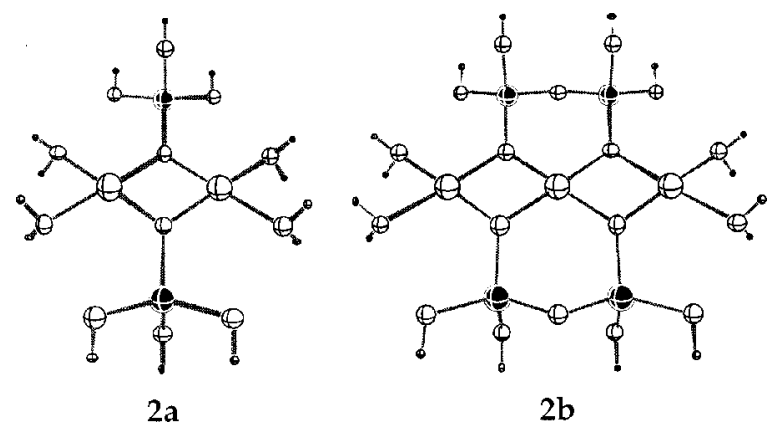

FIG. 2. Molecular models employed to analyze the $J_{c}$ contribution of exchange coupling along the $\mathrm{Cu}_{2} \mathrm{O}_{2}$ chains. In what follows, the two models are called $2 \mathrm{a}$ and $2 \mathrm{~b}$.

the magnetic character with the $\mathrm{Cu}-\mathrm{O}-\mathrm{Cu}$ angle. ${ }^{30}$ Thus, if this angle has a value larger than $98^{\circ}$, the exchange coupling is found to be antiferromagnetic while for smaller values a ferromagnetic behavior shows up. Our theoretical results have shown that actually there is a second structural parameter that also plays a fundamental role: the out-of-plane shift of the atom bonded to the bridging oxygen. ${ }^{24}$ For the hydroxo-bridged complexes, if this atom is located on the $\mathrm{Cu}_{2} \mathrm{O}_{2}$ plane, the calculations predict antiferromagnetic coupling for the whole range of $\mathrm{Cu}-\mathrm{O}-\mathrm{Cu}$ angles. Hence, the strongest antiferromagnetic character is obtained if this atom remains on the $\mathrm{Cu}_{2} \mathrm{O}_{2}$ plane. Large out-of-plane shifts, that appear always combined with smaller $\mathrm{Cu}-\mathrm{O}-\mathrm{Cu}$ angles, result however in a ferromagnetic coupling. A third structural parameter whose influence on the exchange coupling is not negligible, is the $\mathrm{Cu}-\mathrm{O}$ distance. Within the range of experimental values, an increase in the $\mathrm{Cu}-\mathrm{O}$ bond distance results in a stronger antiferromagnetic contribution. ${ }^{25}$ Recently, from inelastic-neutron-scattering experiments and the analysis of the symmetry of the frequency modes, Braden et al. proposed $^{31}$ that the two angular parameters just discussed, the $\mathrm{Cu}-\mathrm{O}-\mathrm{Cu}$ angle and the out-of-plane shift of the germanium atom, are the main factors that control the magnetic coupling in $\mathrm{CuGeO}_{3}$.

In order to apply the theoretical methods previously indicated to the study of the magnetic behavior of $\mathrm{CuGeO}_{3}$, we reduce the dimension of the periodic crystal to a molecular model. Such simplification seems reasonable, given the covalent nature of the bonds present in the structure and the local character of the exchange coupling. Thus, two molecular models have been used [Figs. 2(a) and 2(b)] to study the intrachain coupling, in which the dangling bonds in the terminal oxygen atoms have been saturated by hydrogen atoms.

The values of $J$ calculated for different $\mathrm{Cu}-\mathrm{O}-\mathrm{Cu}$ angles are presented in Fig. $3 .^{32}$ The experimental value of the angle in the high-temperature phase is $99.2^{\circ}$ (see Table I), for which our calculations with the trinuclear model (2b) yield the values $J=-206 \mathrm{~K}$ and $\alpha=0.37$ (i.e., $\alpha J=-76 \mathrm{~K}$ ). These results are in good agreement with the experimental data, taking into account the tiny energy differences involved in the evaluation of $J$ and the errors derived from employing a molecular model for the calculation. By using the simplest model $2 \mathrm{a}$ a too small value of $-60 \mathrm{~K}$ has been obtained for $J$, indicating the need to include at least three copper atoms in the model to accurately reproduce the exchange interac- 


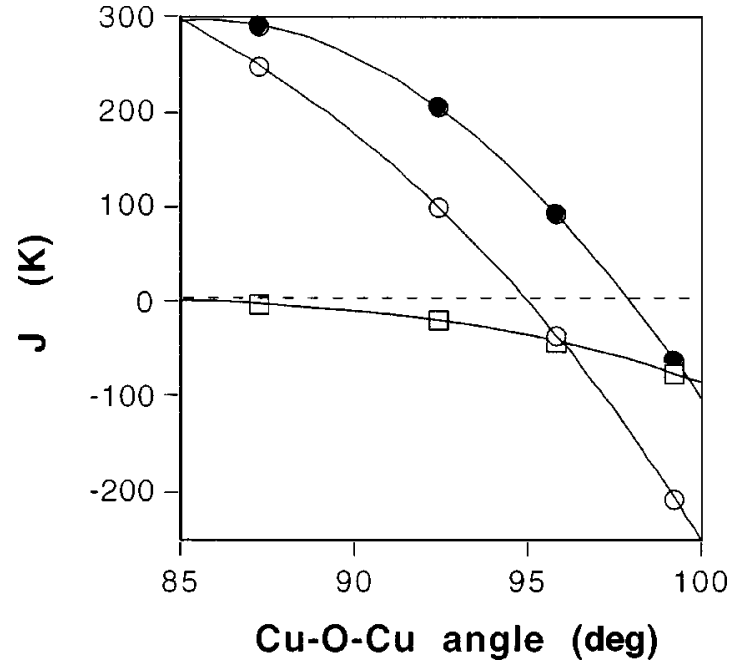

FIG. 3. Calculated exchange coupling values $J$ for model 2a (black circles), $J$ (empty circles), and $\alpha J$ (empty squares) for model $2 \mathrm{~b}$ as a function of the $\mathrm{Cu}-\mathrm{O}-\mathrm{Cu}$ angle.

tions. The substitution in the model of the terminal water ligands by hydroxo-ligands, resulting in an increase of the ionic character of the coordinated oxygen atom, produces a strengthening of the antiferromagnetic coupling close to -80 $\mathrm{K}$.

The calculated variation of the exchange coupling constant with the $\mathrm{Cu}-\mathrm{O}-\mathrm{Cu}$ angle deserves further comments. As expected for the trimer model results, a decrease in that angle results in an increase of $|J|$, with a transition from antiferromagnetic to ferromagnetic coupling at $\mathrm{Cu}-\mathrm{O}-\mathrm{Cu}=95^{\circ}$, amazingly close to the value of $98^{\circ}$ found for the hydroxobridged copper (II) complexes. ${ }^{24}$ Thus, for an angle of $90^{\circ}$ the system should be ferromagnetic in agreement with the predictions of the Goodenough-Kanamori's rules. ${ }^{33-35}$ However, the small distortion, increasing this angle from 90 to $98^{\circ}$, is apparently enough to increase the antiferromagnetic contribution to the coupling, resulting in a change of the magnetic behavior. The analysis of the dependence of $\alpha J$ shows an antiferromagnetic coupling in all the studied range of the $\mathrm{Cu}-\mathrm{O}-\mathrm{Cu}$ angles, vanishing for values under $85^{\circ}$.

To analyze the influence of the second structural parameter, the shift of the Ge atom out of the $\mathrm{Cu}_{2} \mathrm{O}_{2}$ plane, we have repeated our calculations fixing these atoms in such plane while using the experimental $\mathrm{Cu}-\mathrm{O}-\mathrm{Cu}$ angle. Such distortion corresponds to a shift in the $\mathrm{O}-\mathrm{O}-\mathrm{Ge}$ angle of $21.2^{\circ}$. As expected, a stronger antiferromagnetic coupling between nearest neighbors is found for the in-plane configuration, $J=-288 \mathrm{~K}$ and $\alpha J=-74 \mathrm{~K}$, showing a similar tendency to that found for the copper (II) molecular complexes. From these values, one can see that for this case, the exchange coupling is more affected by the $\mathrm{Cu}-\mathrm{O}-\mathrm{Cu}$ angle than by the $\mathrm{O}-\mathrm{O}-\mathrm{Ge}$ one, since in the former case a variation of only $5^{\circ}$ results in a change of more than $-200 \mathrm{~K}$ for $J$. The variation of $J$ with the structural parameters is usually expressed as $\partial J / J \partial \eta$, where $\eta$ are in our case the two angles mentioned above. We obtained a value of 0.26 for the variation of $J$ with the $\mathrm{Cu}-\mathrm{O}-\mathrm{Cu}$ angle. Previously reported values ${ }^{18}$ for this magnitude are strongly model dependent ranging from 0.05 to 0.22 . For the $\mathrm{O}-\mathrm{O}-\mathrm{Ge}$ angle the calculated value is 0.019 , somewhat larger than those given in
Ref. 18 that range between 0.003 to 0.010 .

To analyze the influence of the structural changes associated with the spin-Peierls transition we have calculated the exchange coupling constants for model $2 \mathrm{~b}$, in which the symmetry between neighbor $\mathrm{Cu}$ atoms is broken and each $\mathrm{Cu}_{2} \mathrm{O}_{2}$ moiety adopts one of the structural parameter sets indicated in Table I for the low-temperature phase. Model $2 \mathrm{~b}$ becomes in this case a nonsymmetrical trinuclear system with two $J$ values between nearest neighbors $\left(J_{1}\right.$ and $\left.J_{2}\right)$, and a third coupling constant $J_{3}$ for the coupling between nextnearest neighbors equivalent to $\alpha J$ in the symmetric model. The explicit diagonalization of the spin Hamiltonian for such systems does not provide the three values of $J,^{36}$ and some other simplifying hypothesis must be introduced. A reasonable approximation is for instance to take the same $J_{3}$ value as in the symmetric model. The small variation of $\alpha J$ with the bond angle (Fig. 3) seems to confirm that the small changes introduced in the asymmetric model do not appreciably modify the $\alpha J$ value. With this assumption, values of -134 and $-258 \mathrm{~K}$ are found for $J_{1}$ and $J_{2}$, respectively. The smaller value corresponds to the $\mathrm{Cu}_{2} \mathrm{O}_{2}$ moiety with the smaller $\mathrm{Cu}-\mathrm{O}-\mathrm{Cu}$ angle and the shorter $\mathrm{Cu}-\mathrm{Cu}$ distance, as expected. From these calculated exchange coupling constants a value of 0.30 can be estimated for $\delta$ by taking $\delta=J_{1}$ $-J_{2} / 2 J$. There is a dispersion of $\delta$ values previously reported in the literature (between 0.01 and 0.12) (Refs. 18, 37) depending on the model employed, but our result for this parameter is significantly larger, as expected from the large calculated $\partial J / J \partial \eta$ values discussed previously. The strong dependence of the exchange coupling constants with the $\mathrm{Cu}$ $\mathrm{O}-\mathrm{Cu}$ angle found in Fig. 3, which is also found both experimentally and theoretically for hydroxo- and alkoxo-bridged $\mathrm{Cu}$ (II) binuclear compounds, is however in better agreement with a larger value of $\delta .{ }^{19}$ Using the values of $J_{1}$ and $J_{2}$ corresponding to the symmetric model with the two angles of the distorted structure $\left(98.8\right.$ and $\left.99.6^{\circ}\right)$ a $\delta$ value of 0.12 has been obtained. This value is logically lower than the previous one since only the effect of the $\mathrm{Cu}-\mathrm{O}-\mathrm{Cu}$ angle has been considered and it is in agreement with the largest $\delta$ value previously estimated. ${ }^{18,38}$ In this approximation, both $\delta$ values $(0.30$ and 0.12$)$ indicate, however, a stronger dimerization of the exchange coupling in the chains than the values previously reported.

\section{CONCLUSIONS}

We have studied the magnetostructural correlation for the spin-Peierls compound $\mathrm{CuGeO}_{3}$ by using density-functional calculations for molecular models. The coupling constants obtained using the symmetric trinuclear model are close to those obtained from experimental measurements. A dinuclear model provides too small values for the exchange antiferromagnetic coupling constant. Considering the variation of $J$ with the $\mathrm{Cu}-\mathrm{O}-\mathrm{Cu}$ angle and the shift of the germanium atom out of the $\mathrm{Cu}_{2} \mathrm{O}_{2}$ plane, we have found a stronger dependence of the exchange coupling on the $\mathrm{Cu}-\mathrm{O}-\mathrm{Cu}$ angle than with the out-of-plane shift of the Ge atom. The calculated trends are similar to the ones obtained experi- 
mentally for hydroxo- and alkoxo-bridged $\mathrm{Cu}$ (II) binuclear complexes and to those predicted in our previous theoretical results. Thus, an increase of the $\mathrm{Cu}-\mathrm{O}-\mathrm{Cu}$ angle causes a stronger antiferromagnetic coupling while the out-of-plane shift of the germanium atom reduces the antiferromagnetic behavior. This strong dependence of the exchange coupling with the structural parameters, leads to larger values for dimerization parameter $\delta$ than those previously reported in the literature.

\section{ACKNOWLEDGMENTS}

Access to the computing resources of the Centre de Supercomputació de Catalunya (CESCA) was possible thanks in part to a grant from Comissió Interdepartomental de Recerca $i$ Innovacio Tecnologica (CIRIT) and the Universitat de Barcelona. Financial support for this work was provided by DGES (Grant No. PB95-0848-C02-01) and by the European Science Foundation (Molecular Magnets programme).
${ }^{1}$ J. W. Bray, L. V. Interrante, L. S. Jacobs, and J. C. Bonner, in Extended Linear Chain Compounds, edited by J. S. Miller (Plenum, New York, 1983), Vol. 3.

${ }^{2}$ S. Huizinga, J. Kommandeur, G. A. Sawatzy, B. T. Thole, K. Kopinga, W. J. de Jonge, and J. Roos, Phys. Rev. B 19, 4723 (1979).

${ }^{3}$ M. Hase, I. Terasaki, and K. Uchinokura, Phys. Rev. Lett. 70, 3651 (1993).

${ }^{4}$ J. P. Boucher and L. P. Regnault, J. Phys. I 6, 1939 (1996).

${ }^{5}$ H. Völlenke, A. Wittman, and N. Nowotny, Monatsch. Chem. 98, 1352 (1967).

${ }^{6}$ K. Hirota, D. E. Cox, J. E. Lorenzo, G. Shirane, J. M. Tranquada, M. Hase, K. Uchinokura, H. Kojima, Y. Shibuya, and I. Tanaka, Phys. Rev. Lett. 73, 736 (1994)

${ }^{7}$ J. P. Pouget, L. P. Regnault, M. Ain, B. Hennion, J. P. Renard, P. Veillet, G. Dhaleene, and A. Revcolevschi, Phys. Rev. Lett. 72, 4037 (1994).

${ }^{8}$ M. Hidaka, M. Hatae, I. Yamada, M. Nishi, and J. Akimitsu, J. Phys.: Condens. Matter 9, 809 (1997).

${ }^{9}$ O. Kamimura, M. Terauchi, M. Tanaka, O. Fujita, and J. Akimitsu, J. Phys. Soc. Jpn. 63, 2467 (1994).

${ }^{10}$ M. Isobe and Y. Ueda, J. Phys. Soc. Jpn. 65, 1178 (1996).

${ }^{11}$ H. G. von Schnering, Y. Grin, M. Kaupp, M. Somer, R. K. Kremer, O. Jepsen, T. Chatterji, and M. Weiden, Z. Kristallogr. 213, 246 (1998).

${ }^{12}$ J. Lüdecke, A. Jobst, S. van Smaalen, E. Morré, C. Geibel, and H.-G. Krane, Phys. Rev. Lett. 82, 3633 (1999).

${ }^{13}$ M. Nishi, O. Fujita, and J. Akimitsu, Phys. Rev. B 50, 6508 (1994).

${ }^{14}$ J. Riera and A. Dobry, Phys. Rev. B 51, 16098 (1995).

${ }^{15}$ G. Castilla, S. Chakravarty, and V. J. Emery, Phys. Rev. Lett. 75, 1823 (1995).

${ }^{16}$ K. Fabricius, A. Klümper, U. Löw, B. Büchner, T. Lorenz, G. Dhalenne, and A. Revcolevschi, Phys. Rev. B 57, 1102 (1998).

${ }^{17}$ H. Nojiri, Y. Shimamoto, N. Miura, M. Hase, K. Uchinokura, H. Kojima, I. Tanaka, and Y. Shibuya, Phys. Rev. B 52, 12749 (1995).

${ }^{18}$ R. Werner, C. Gros, and M. Braden, Phys. Rev. B 59, 14356 (1999).

${ }^{19}$ Z. S. Popovic, F. R. Vukajlovic, and Z. V. Slijvancanin, J. Phys.: Condens. Matter 7, 4549 (1995).

${ }^{20}$ L. F. Mattheiss, Phys. Rev. B 49, 14050 (1994).
${ }^{21}$ Z. V. Sljivancanin, Z. S. Popovic, and F. R. Vukajlovic, Phys. Rev. B 56, 4432 (1997).

${ }^{22}$ S. Zagoulaev and I. I. Tupitsyn, Phys. Rev. B 55, 13528 (1997).

${ }^{23}$ J. Cano, P. Alemany, S. Alvarez, E. Ruiz, and M. Verdaguer, Chem. Eur. J. 4, 476 (1998).

${ }^{24}$ E. Ruiz, P. Alemany, S. Alvarez, and J. Cano, J. Am. Chem. Soc. 119, 1297 (1997).

${ }^{25}$ E. Ruiz, P. Alemany, S. Alvarez, and J. Cano, Inorg. Chem. 36, 3683 (1997).

${ }^{26}$ A. D. Becke, J. Chem. Phys. 98, 5648 (1993).

${ }^{27}$ Gaussian94: M. J. Frisch, G. W. Trucks, H. B. Schlegel, P. M. W. Gill, B. G. Johnson, M. A. Robb, J. R. Cheeseman, T. A. Keith, G. A. Petersson, J. A. Montgomery, K. Raghavachari, M. A. Al-Laham, V. G. Zakrzewski, J. V. Ortiz, J. B. Foresman, J. Cioslowski, B. B. Stefanov, A. Nanayakkara, M. Challacombe, C. Y. Peng, P. Y. Ayala, W. Chen, M. W. Wong, J. L. Andres, E. S. Replogle, R. Gomperts, R. L. Martin, D. J. Fox, J. S. Binkley, D. J. Defrees, J. Baker, J. J. P. Stewart, M. HeadGordon, C. Gonzalez, and J. A. Pople (Gaussian, Inc, Pittsburgh, PA, 1994).

${ }^{28}$ A. Schaefer, C. Huber, and R. Ahlrichs, J. Chem. Phys. 100, 5829 (1994).

${ }^{29}$ A. Schaefer, H. Horn, and R. Ahlrichs, J. Chem. Phys. 97, 2571 (1992).

${ }^{30}$ V. H. Crawford, H. W. Richardson, J. R. Wasson, D. J. Hodgson, and W. E. Hatfield, Inorg. Chem. 15, 2107 (1976).

${ }^{31}$ M. Braden, B. Hennion, W. Reichardt, G. Dhalenne, and A. Revcolevschi, Phys. Rev. Lett. 80, 3634 (1998).

${ }^{32}$ The exchange coupling constants for a trinuclear system $A B A$ have been determined using the vectorial approach $\left(S^{\prime}=S_{A 1}\right.$ $+S_{A 2}$ and $\left.S=S^{\prime}+S_{B}\right)$. Thus, the relative energies $E\left(S, S^{\prime}\right)$ for the three states are $E(1 / 2,1)=0, E(3 / 2,1)=-3 J / 2$, and $E(1 / 2,0)=-J(1-\alpha)$. For the dimer model, the value $J$ is directly the singlet-triplet energy gap.

${ }^{33}$ J. Kanamori, J. Phys. Chem. Solids 10, 87 (1959).

${ }^{34}$ J. B. Goodenough, Magnetism and Chemical Bond (Interscience, New York, 1963).

${ }^{35}$ J. B. Goodenough, Phys. Rev. 100, 564 (1955).

${ }^{36}$ O. Kahn, Molecular Magnetism (VCH, New York, 1993).

${ }^{37}$ B. Büchner, H. Fehske, A. P. Kampf, and G. Wellein, Physica B 259, 956 (1999).

${ }^{38}$ W. Brenig, Phys. Rev. B 56, 14441 (1997). 